J. Phys. B: At. Mol. Opt. Phys.,39, (2006) p.1863-1877.

ISSN (Print): 0953-4075 ISSN (Online): 1361-6455

doi:10.1088/0953-4075/39/8/005

(C) IOP Publishing Ltd

This is an un-copyedited version of an article accepted for publication in J. Phys. B: At. Mol. Opt. Phys. IOP

Publishing Ltd is not responsible for any errors or omissions in this version of the manuscript or any version derived from it. The definitive publisher authenticated version is available online at doi:10.1088/0953-4075/39/8/005.

http://journals.iop.org/

http://www.iop.org/EJ/journal/JPhysB

\title{
Relativistic and correlation effects in electron impact excitation of forbidden transitions of OII
}

\author{
Maximiliano Montenegro $^{1,2}$, Werner Eissner ${ }^{3}$, Sultana N Nahar ${ }^{1}$ and Anil K Pradhan ${ }^{1}$ \\ ${ }^{1}$ Department of Astronomy, The Ohio State University, Columbus, OH 43210, USA \\ ${ }^{2}$ Department of Education, The Ohio State University, Columbus, OH 43210, USA \\ ${ }^{3}$ Institut für Theoretische Physik, Teilinstitut 1, 70550 Stuttgart, Germany
}

\begin{abstract}
We investigate relativistic and correlation effects in electron impact excitation of singly ionized oxygen using the Breit-Pauli $R$-matrix method. The intermediate coupling close-coupling calculations are carried out using a 16-level target representation dominated by the electronic configurations $1 s^{2} 2 s^{2} 2 p^{3}, 1 s^{2} 2 s 2 p^{4}, 1 s^{2} 2 s^{2} 2 p^{2} 3 s$. Resonance structures are delineated in detail to ascertain the effect on averaged collision strengths. Convergence of the partial wave summation is ensured for non-dipole transitions in the $R$-matrix calculations. The present results differ significantly from the similar Breit-Pauli $R$-matrix calculations by McLaughlin and Bell (1998 J. Phys. B: At. Mol. Opt. Phys. 31 4317-29), but are essentially in agreement with the $L S$ coupling results of Pradhan (1976a J. Phys. B: At. Mol. Opt. Phys. 9 433-43, 1976b Mon. Not. R. Astron. Soc. 177 31-8). A comprehensive study of the detailed energy behaviour of all forbidden transitions among the five levels of the ground configuration, i.e. $2 s 2 p^{3}\left({ }^{4} S_{3 / 2}^{O},{ }^{2} D_{5 / 2}^{0}{ }_{3 / 2},{ }^{2} P_{3 / 2}^{0}\right.$, ${ }_{1} 2$ ) shows that the finestructure collision strengths do not significantly depart from the values obtained from a purely $L S \rightarrow L S J$ transformation, and relativistic effects are therefore small. We find that the Maxwellian-averaged effective collision strengths for the ten transitions also differ from the previous work, most likely due to more extensive delineation of resonances in the present work. However, the differences are largely systematic and therefore the OII line intensity ratios are not significantly affected. We also obtain an excellent agreement between the present-calculated cross sections for the ${ }^{4} S^{o}-{ }^{2} D^{o}$ transition and the experimental merged beam measurements.
\end{abstract}

\section{Introduction}

Because of its cosmic abundance and atomic properties all ions of oxygen are of importance in various astrophysical sources. Singly ionized oxygen is a prominent constituent of gaseous nebulae as a primary diagnostic of electron density (e.g. Seaton and Osterbrock 1957, Osterbrock 1989, Dopita and Sutherland 2003). The pair of lines due to forbidden transitions within the ground configuration of $1 s^{2} 2 s^{2} 2 p^{3}$ of OII (denoted as [OII]) are ${ }^{4} S_{3 / 2}^{o} \rightarrow{ }^{2} \mathrm{D}_{5 / 2,3 / 2}^{0}$ that lie towards the blue end of the optical spectrum at wavelengths $3729 \AA$ and $3726 \AA$. But despite considerable efforts devoted to high-precision atomic calculations for oxygen ions, many of the atomic parameters are known with insufficient accuracy. Since the ground configuration metastable levels are low-lying in energy, they can be readily excited by electron impact at ambient temperatures $T \approx$ 
$10^{4} \mathrm{~K}$ prevalent in most gaseous regions ionized by hot stars, such as the central stars of planetary nebulae or diffuse nebulae such as the Orion created by ionization of a giant molecular cloud by hot young O-stars. Because of their importance, and to resolve outstanding discrepancies in collision strengths for these two ions from the previous-distorted wave calculations, among the first calculations to be carried out with the then new close-coupling codes were for [OII] transitions (Pradhan 1976a, 1976b) (hereafter P76a,b). Due to computational constraints those CC calculations were in $L S$ coupling and limited to the first five terms $2 \mathrm{~s}^{2} 2 \mathrm{p}^{3}\left({ }^{4} \mathrm{~S}^{\mathrm{o}},{ }^{2} \mathrm{D}^{\mathrm{o}},{ }^{2} \mathrm{P}^{\mathrm{o}}\right), 2 \mathrm{~s} 2 \mathrm{p}^{4}\left({ }^{4} \mathrm{P}\right.$, $\left.{ }^{2} \mathrm{D}\right)$. Nevertheless, an algebraic transformation of reactance matrices yielded finestructure collision strengths which, in turn, gave line intensity ratios in good agreement with observations (Wang et al 2004). In addition the forbidden line [OII] ratios gave electron densities consistent with [SII] lines, which lie at the red end of the optical spectrum and originate in the similar atomic structure of SII with a $3 \mathrm{p}^{3}$ open-shell ground configuration.

The main point concerns the low-density limit, where every excitation from the ground state ${ }^{4} S_{3 / 2}$ to the ${ }^{2} D_{5 / 2}^{O} 3 / 2$ levels is followed by radiative decay; collisional mixing between the excited ${ }^{2} D_{J}^{o}$ levels can be neglected, since the population of the excited levels is extremely low and the mixing rate, proportional to the product of ion density in excited levels and electron density, is negligible. Under such conditions, the low-density line intensity ratio reads

$$
\lim _{N_{\mathrm{e}} \rightarrow 0} \frac{I(3729)}{I(3726)}=\frac{q\left({ }^{4} \mathrm{~S}_{3 / 2}^{\circ}-{ }^{2} \mathrm{D}_{5 / 2}^{\circ}\right)}{q\left({ }^{4} S_{3 / 2}^{\circ}-{ }^{2} \mathrm{D}_{3 / 2}^{\circ}\right)},
$$

where the excitation rate coefficient $q_{i j}$ is

$$
q_{i j}(T)=\frac{8.63 \times 10^{-6} \mathrm{~cm}^{3} \mathrm{~s}^{-1} \exp \left(-E_{i j} / k T\right)}{g_{i} \sqrt{T / \mathrm{K}}} \Upsilon_{i j}(T)
$$

with $g_{i}$ being the statistical weight of the initial level and being the $Y_{i j}$ Maxwellian-averaged collision strength

$$
\Upsilon_{i j}(T)=\int_{E_{i}}^{\infty} \Omega_{i j}(E) \exp (-E / k T) \mathrm{d}(E / k T)
$$

A purely algebraic transformation from the $L S$ coupling to a pair-coupling representation may be employed to obtain finestructure $L S J$ collision strengths, which should be accurate provided the relativistic effects are negligible. The transformation coefficients are particularly simple when the initial $L S$ term has $L$ or $S=0$, and

$$
\frac{\Omega\left(S L J-S^{\prime} L^{\prime} J^{\prime}\right)}{\Omega\left(S L-S^{\prime} L^{\prime}\right)}=\frac{\left(2 J^{\prime}+1\right)}{\left(2 S^{\prime}+1\right)\left(2 L^{\prime}+1\right)}
$$

This procedure was employed by P76a,b using the IMPACT close-coupling codes (Eissner and Seaton 1972, 1974, Crees et al 1978). It is then clear that in the low-density limit

$$
\lim _{N_{e} \rightarrow 0} \frac{I\left({ }^{4} S_{3 / 2}^{o}-{ }^{2} \mathrm{D}_{5 / 2}^{\circ}\right)}{I\left({ }^{\circ} S_{3 / 2}^{o}-{ }^{2} \mathrm{D}_{3 / 2}^{\circ}\right)}=1.5 .
$$


But the above analysis is predicated on the assumption that $L S$ coupling is valid and there is no deviation due to relativistic effects.

On the other hand, relativistic effects involving the Einstein coefficients $A$ for forbidden spontaneous radiative transitions are crucial in determining the high-density limit, where the OII line intensity ratio is given by the ratio of the transition probabilities

$$
\lim _{N_{e} \rightarrow \infty} \frac{I\left({ }^{4} S_{3 / 2}^{o}-{ }^{2} \mathrm{D}_{5 / 2}^{o}\right)}{I\left({ }^{4} S_{3 / 2}^{o}-{ }^{2} \mathrm{D}_{3 / 2}^{o}\right)}=\frac{6}{4} \frac{A\left({ }^{2} \mathrm{D}_{5 / 2}^{o}-{ }^{4} \mathrm{~S}_{3 / 2}^{o}\right)}{A\left({ }^{2} \mathrm{D}_{3 / 2}^{o}-{ }^{4} \mathrm{~S}_{3 / 2}^{o}\right)}=0.35
$$

The transition probabilities $A$ for these predominantly magnetic dipole (M1) transitions were computed accurately by Eissner and Zeippen (1981) and Zeippen (1982). Eissner and Zeippen, in fact, obtained the high-density limit of this ratio by not only using configuration-mixing-type expansions, but also including relativistic terms of order $\alpha^{2} Z^{2}$ and $\alpha^{2} Z$ to the first-order M1 operator, based on the previous work by Drake (1971). Thus both the low- and the high-density theoretical limits are fixed by statistics and the atomic structure alone. Bell and Hibbert (1990) also demonstrated that departures from $L S$ coupling occur for several transition probabilities.

Following Bell and Hibbert's (1990) calculations, McLaughlin and Bell (1998, MB89) embarked on a much larger calculation than P76a,b to investigate relativistic effects in the collision strengths using the Breit-Pauli $R$-matrix (hereafter BPRM) method. It came as a tremendous surprise that the finestructure collision strengths differed substantially from those of P76a,b, apparently due to departures from $L S$ coupling, a more extensive close-coupling expansion, and better resolution of resonances. Keenan et al (1999) used the MB98 results to calculate, in particular, the [OII] I (3729)/I (3726) line intensity ratio, which was $\approx 2.0$, about $30 \%$ higher than the non-relativistic $L S$ coupling low-density limit of 1.5. Subsequently, however, several observational studies of gaseous nebulae found that the MB98 results are not consistent with observations (Copetti and Writzl 2002, Wang et al 2004), and that the earlier P76a,b results were to be preferred.

In this paper we therefore undertake new calculations, also using the BPRM method, and a different target expansion, to ascertain the relevant [OII] collision strengths with high accuracy and the source of the discrepancy with the MB98 results.

\section{Formulation}

Close-coupling (CC) calculations using the $R$-matrix method (Burke et al 1971, Seaton 1987) have been described in many previous publications. As the emphasis of this work is to investigate relativistic and correlation effects together, we briefly outline the BPRM approach as employed herein.

The OII target wavefunctions are calculated using superstructure (Eissner and Seaton 1974), which employs a scaled Thomas-Fermi-Dirac-Amaldi potential (Eissner and Nussbaumer 1969) to compute a set of one-electron orbitals; the scaling parameters optimize the unweighted sum over non-relativistic terms' energies arising from a set of spectroscopic configurations. With such radial functions, the Breit-Pauli Hamiltonian (Berrington et al 1995) (including two-body magnetic terms) yields the level energies $E_{i}$. For a target so specified electron collision processes are addressed in a partial wave expansion with radial functions satisfying the Breit-Pauli Hamiltonian obtaining close-coupling (CC) solutions with the Breit-Pauli $R$-matrix (BPRM) code 
(Scott and Taylor 1982).

$$
H_{N+1}^{\mathrm{BP}}=H_{N+1}+H_{N+1}^{\mathrm{mass}}+H_{N+1}^{\mathrm{Dar}}+H_{N+1}^{\mathrm{so}},
$$

In this equation $H_{N+1}$ is the non-relativistic Hamiltonian along with the one-body mass-velocity term, the Darwin term and the spin-orbit term. The two-body terms

in the BP Hamiltonian (discussed in the following section) are not considered, and therefore the current form of the BPRM method amounts to reducing the Dirac equation to Pauli form and dropping two-body electron-electron contributions of the BP order. The spin-orbit term breaks the $L S$ symmetry, leading to finestructure levels $J \pi$ of the total angular momentum quantum number $J$ at parity $\pi$; mass and Darwin terms are retained and provide additional energy scaling due to relativistic effects.

The partial wave expansion for the (e+ion) $(N+1)$-electron system is described by wavefunctions $\Psi(E)$ of a total spin and the angular symmetry $S L \pi$ or $J \pi$ and represented as products of the target ion states and partial waves:

$$
\Psi(E)=A \sum_{i} \chi_{i} \theta_{i}+\sum_{j} c_{j} \Phi_{j}
$$

where $\chi i$ is the target wavefunction of a specific term $S_{i} L_{i} \pi_{i}$ or level $J_{i} \pi_{i}$, and $\theta i$ is the wavefunction of the colliding electron $(N+1)$ in a channel labelled $S_{l} L_{l}\left(J_{l}\right) \pi_{i} k_{l} l_{l}(S L \pi)(J \pi)$; the quantity $k_{l}$ is the channel wave number, hence $X_{l}=\left(k_{l} a_{0}\right)^{2}$ Ry asymptotically the associated kinetic energy $(<0$ if channel closed). Symbols $\Phi_{j}$ mark correlation wavefunctions made up of $(N+1)$ bound electrons to (a) compensate for the orthogonality conditions imposed on continuum with bound orbitals for computational convenience, (b) represent additional short-range correlations that are often of crucial importance in scattering and radiative $\mathrm{CC}$ calculations. They are sometimes referred to as 'bound channels', as opposed to the continuum or 'free' channels in the sum over the target states $\chi_{i}$. In relativistic BPRM calculations' sets of the collisional symmetry, $S L \pi$ are coupled to obtain (e + ion) states with total $J \pi$, followed by diagonalization of the $(N+1)$-electron Hamiltonian. Details of diagonalizing $H$ at the $R$-matrix boundary are given in the published work (e.g. Berrington et al 1995); so is its outward propagation.

It is important to satisfy a number of criteria in CC calculations: (A) accuracy of target eigenfunctions, (B) completeness of partial wave expansions, (C) enough target levels in the free-channel (open or closed) first term in (8), (D) consistency between the first free-channel term and the second bound-channel correlation terms. Furthermore, there are other criteria that need to be met, such as adequate resolution of resonances particularly in the near-threshold region, and correct high-energy behaviour particularly for dipole transitions. The accuracy of the CC calculations should therefore not be judged only by the number of target levels included, but by all of the above.

The measurable quantity is the (excitation) cross section $\mathrm{o}_{\mathrm{ij}}$, which is related to the collision strength by

$$
\sigma_{i j}\left(X_{i}\right)=\pi \frac{\Omega_{i j}\left(X_{1}\right)}{g_{i} k_{i}^{2}} \equiv \frac{\Omega_{i j}\left(X_{1}\right)}{g_{i}\left(k_{i} a_{0}\right)^{2}} \pi a_{0}^{2},
$$

where $g_{i}$ is the statistical weight $\left(2 J_{i}+1\right)$ and $X i=\left(k_{i} a_{0}\right)^{2}$ Ry the large distance kinetic energy of an 
electron associated with the initial state $i$ (n.b. save finite mass correction $1 \mathrm{Ry} \approx 13.6 \mathrm{eV}$ is the non-relativistic ionization energy of neutral hydrogen, $\pi a_{0}^{2}$ is the hydrogenic ground state area, $E_{i}+$ $X_{i}$ is invariant at specified collision energies $X_{l}$ ).

\section{Computations}

In our close-coupling approach to electron impact excitation, we retain the lowest 16 target levels of OII, i.e. eight terms arising from the three spectroscopic configurations $2 s^{2} 2 p^{3}, 2 s 2 p^{4}$ and $2 s^{2} 2 p^{2} 3 s$ (dropping the $1 \mathrm{~s}^{2}$ closed shell from the notation), and we allow for term mixing among the entire complex of nine configurations. In their reference calculation of

Table 1. Levels and energies of the OII target-1-magn: without magnetic interactions between valence shell electrons, 2-magn: calculation including all finestructure terms of the Breit-Pauli order.

\begin{tabular}{|c|c|c|c|c|c|c|c|}
\hline \multirow{2}{*}{$\begin{array}{l}\text { level } \\
\text { index }\end{array}$} & & & \multirow{2}{*}{$\begin{array}{l}E^{\mathrm{STO}} / \mathrm{Ry} \\
\mathrm{McL} \& \mathrm{~B}\end{array}$} & \multirow{2}{*}{$\begin{array}{l}\mathcal{E}^{\text {calc }} / \text { Ry } \\
2 \text {-magn }\end{array}$} & \multicolumn{2}{|c|}{$E^{\text {calc }} / \mathrm{Ry}$} & \multirow{2}{*}{$\begin{array}{l}E^{\mathrm{obs}} / \mathrm{Ry} \\
\text { Wenåker }\end{array}$} \\
\hline & \multicolumn{2}{|c|}{ designation } & & & 1-magn & 2-magn & \\
\hline 1 & \multirow[t]{5}{*}{$2 s^{2} 2 p^{3}$} & ${ }^{4} S_{3 / 2}^{\circ}$ & 0 & 0 & 0 & 0 & 0 \\
\hline 2 & & ${ }^{2} \mathrm{D}_{5 / 2}^{\mathrm{o}}$ & 0.24789 & 0.28238 & 0.27370 & 0.27360 & 0.24432 \\
\hline 3 & & ${ }^{2} \mathrm{D}_{3 / 2}^{\mathrm{o}}$ & 0.24791 & 0.28258 & 0.27366 & 0.27381 & 0.24450 \\
\hline 4 & & ${ }^{2} \mathrm{P}_{3 / 2}^{\mathrm{o}}$ & 0.37413 & 0.38211 & 0.36976 & 0.36973 & 0.36877 \\
\hline 5 & & ${ }^{2} \mathrm{P}_{1 / 2}^{\mathrm{o}}$ & 0.37410 & 0.38219 & 0.36972 & 0.36978 & 0.36879 \\
\hline 6 & \multirow[t]{6}{*}{$2 s 2 p^{4}$} & ${ }^{4} \mathrm{P}_{5 / 2}$ & 1.12351 & 1.10392 & 1.09128 & 1.09122 & 1.09204 \\
\hline 7 & & ${ }^{4} \mathrm{P}_{3 / 2}$ & 1.12480 & 1.10548 & 1.09269 & 1.09276 & 1.09353 \\
\hline 8 & & ${ }^{4} \mathrm{P}_{1 / 2}$ & 1.12456 & 1.10628 & 1.09353 & 1.09356 & 1.09428 \\
\hline \multirow[t]{2}{*}{9} & & ${ }^{2} \mathrm{D}_{5 / 2}$ & 1.54667 & 1.61946 & 1.61037 & 1.61030 & \\
\hline & & & & & & [1.610 10] & 1.51260 \\
\hline 10 & & ${ }^{2} \mathrm{D}_{3 / 2}$ & 1.54660 & 1.61949 & 1.61023 & $\begin{array}{l}1.61029 \\
{[1.61019]}\end{array}$ & 1.51267 \\
\hline 11 & \multirow[t]{5}{*}{$2 s^{2} 2 p^{2}\left({ }^{3} P\right) 3 s$} & ${ }^{4} \mathrm{P}_{1 / 2}$ & 1.67762 & 1.72883 & 1.77525 & 1.77539 & 1.68799 \\
\hline 12 & & ${ }^{4} \mathrm{P}_{3 / 2}$ & 1.67859 & 1.72971 & 1.77609 & 1.77618 & 1.68895 \\
\hline 13 & & ${ }^{4} \mathrm{P}_{5 / 2}$ & 1.68021 & 1.73104 & 1.77751 & 1.77740 & 1.69039 \\
\hline 14 & & ${ }^{2} \mathrm{P}_{1 / 2}$ & 1.72595 & 1.80867 & 1.81643 & 1.81655 & 1.72128 \\
\hline 15 & & ${ }^{2} \mathrm{P}_{3 / 2}$ & 1.72778 & 1.81025 & 1.81811 & 1.81805 & 1.72292 \\
\hline 16 & $2 s 2 p^{4}$ & ${ }^{2} \mathrm{~S}_{1 / 2}$ & 1.83618 & 1.87420 & 1.87161 & 1.87161 & 1.78344 \\
\hline 17 & \multirow[t]{5}{*}{$2 s^{2} 2 p^{2}\left({ }^{3} P\right) 3 p$} & ${ }^{2} \mathrm{~S}_{1 / 2}^{\mathrm{o}}$ & - & & & [1.94078] & 1.85846 \\
\hline 18 & & ${ }^{4} \mathrm{D}_{1 / 2}^{\mathrm{O}}$ & - & & & [1.963 40] & 1.88387 \\
\hline 19 & & ${ }^{4} \mathrm{D}_{3 / 2}^{\mathrm{o}}$ & - & & & [1.963 82] & 1.88437 \\
\hline 20 & & ${ }^{4} \mathrm{D}_{5 / 2}^{\mathrm{o}}$ & - & & & [1.96451] & 1.88521 \\
\hline 21 & & ${ }^{4} \mathrm{D}_{7 / 2}^{\mathrm{o}}$ & - & & & [1.965 45] & 1.88634 \\
\hline 22 & \multirow[t]{2}{*}{$2 s^{2} 2 p^{2}\left({ }^{1} D\right) 3 s$} & ${ }^{2} \mathrm{D}_{5 / 2}$ & 1.90172 & & & [1.971 58] & 1.88606 \\
\hline 23 & & ${ }^{2} \mathrm{D}_{3 / 2}$ & 1.90173 & & & [1.971 59] & 1.88607 \\
\hline$\vdots$ & \multirow{3}{*}{$\begin{array}{c}: \\
2 \mathrm{~s} 2 \mathrm{p}^{4}\end{array}$} & & - & & & $\vdots$ & $\vdots$ \\
\hline 32 & & ${ }^{2} \mathrm{P}_{3 / 2}$ & 2.08127 & & & [2.085 29] & 1.93730 \\
\hline 33 & & ${ }^{2} \mathrm{P}_{1 / 2}$ & 2.08283 & & & {$[2.08680]$} & 1.93883 \\
\hline 34 & \multirow[t]{4}{*}{$2 s^{2} 2 p^{2}\left({ }^{1} D\right) 3 p$} & ${ }^{2} \mathrm{~F}_{5 / 2}^{\mathrm{o}}$ & - & & & [2.16294] & 2.08428 \\
\hline 35 & & ${ }^{2} \mathrm{~F}_{7 / 2}^{\mathrm{o}}$ & - & & & [2.163 10] & 2.08450 \\
\hline 36 & & ${ }^{2} \mathrm{D}_{5 / 2}^{\mathrm{o}}$ & - & & & [2.20067] & 2.09543 \\
\hline 37 & & ${ }^{2} \mathrm{D}_{3 / 2}^{\mathrm{o}}$ & - & & & [2.20095] & 2.09563 \\
\hline 38 & $2 s^{2} 2 p^{2}\left({ }^{1} S\right) 3 s$ & ${ }^{2} S_{1 / 2}$ & 2.17157 & & & [2.23792] & 2.10147 \\
\hline
\end{tabular}

Note - apart from the three spectroscopic configurations the following six correlation configurations are involved: $2 s^{2} 2 p^{2} 3 p, 2 s^{2} 2 p^{2} 3 d, 2 p^{5}, 2 s 2 p^{3} 3 s, 2 s 2 p^{3} 3 p$ and $2 s 2 p^{3} 3 d$.

1998, McLaughlin and Bell (McL\&B) add the remaining five levels that arise from $2 s 2 p^{4}$ and 
$2 s^{2} 2 p^{2} 3 s$. They are embedded in states associated with 'correlation' configurations in table 1observed values are extracted from Wenåker (1990) up to $2 \mathrm{~s}^{2} 2 \mathrm{p}^{2}\left({ }^{1} \mathrm{~S}\right) 3 \mathrm{~s}{ }^{2} \mathrm{~S}$. It looks like a straight adaptation from the 11-term target employed by Bell et al (1989) for photoionizing OII in $L S$ coupling to intermediate coupling. We require a target with an energetically contiguous set of levels. As we focus on the 'forbidden' transitions between the five levels of the ground configuration, resonances converging on levels beyond $\approx 1.8$ Ry contribute only marginally to the Maxwellian-damped rate coefficients at temperatures of interest; for example, at $\mathrm{T} \approx 10^{4} \mathrm{~K}$

Table 2. Properties of the OII target radial orbital functions.

\begin{tabular}{|c|c|c|c|c|c|c|}
\hline \multirow[b]{2}{*}{$n l$} & \multirow[b]{2}{*}{$\lambda$} & \multirow[b]{2}{*}{$r_{\text {cut }} / a_{0}$} & \multicolumn{2}{|c|}{$\bar{r} / a_{0}$} & \multirow{2}{*}{$\begin{array}{l}r_{\text {inff }} / a_{0} \\
\text { last }\end{array}$} & \multirow{2}{*}{$\begin{array}{l}P(\mathrm{RA}) \\
\left.\text { (at } 17 a_{0}\right)\end{array}$} \\
\hline & & & Superstructure & BPRM & & \\
\hline 1s & 1.350 & 3.657 & 0.19911 & 0.19981 & $2.5 \times 10^{-1}$ & $<0.129 \times 10^{-29}$ \\
\hline $2 s$ & 1.250 & 3.379 & 1.05078 & 1.05078 & 1.3 & $-0.866 \times 10^{-12}$ \\
\hline $2 p$ & 1.120 & 3.101 & 1.12267 & 1.12267 & 1.4 & $+0.106 \times 10^{-08}$ \\
\hline $3 \mathrm{~s}$ & 1.070 & 3.379 & 3.79028 & 3.79029 & 4.9 & $+0.148 \times 10^{-03}$ \\
\hline $3 p$ & 1.050 & 4.027 & 4.42821 & 4.42819 & 5.8 & $-0.932 \times 10^{-03}$ \\
\hline $3 \mathrm{~d}$ & 1.100 & 4.397 & 5.07610 & 5.07563 & 6.9 & $+0.503 \times 10^{-02}$ \\
\hline
\end{tabular}

the exponential damping factor in equation (3) is $\sim \exp (-16)$ at $E=1 \mathrm{Ry}$. This is borne out by the present results described in the following section, which basically agree with the much older P76a,b calculations done on a smaller scale.

Table 2 summarizes the properties of the statistical model radial functions $P_{n l}(r)$ employed for the target expansion in superstructure, leading to level energies shown in the two $E^{\text {calc }}$ columns in table 1. Column $\varepsilon^{\text {calc }}$ shows rather unsatisfactory results from optimizing the unweighted energy sum over the first eight terms of table 1 , when the scaling factors $\lambda_{n l}$ become 1.483 37, 1.21267, 1.167 64, and (because otherwise the flat functional would not converge) for $3 \mathrm{~s}, 3 \mathrm{p}$ and $3 \mathrm{~d} 1.488$ 64 in the order of table 2, with associated mean radii of $0.19829,1.06604,1.07921,3.22548$, 3.56671 and 4.59529 Bohr radii $a 0$. Other functionals derived from the nine configurations are even less satisfactory. We therefore resort to some hybrid optimization. Starting with a smaller value $\lambda_{1 \mathrm{~s}}=1.350$ mitigates against overvariation in varying $\lambda_{l}$ independently for different $n$ (and the subsequent Schmidt orthogonalization), while there are no additional high- $n$ correlation configurations; we mention that $\lambda_{s}=1.2248$ results from a calculation that scales each set of one orbitals with the same variational parameter.

Rather convincing is the finestructure splitting with our choice of orbitals. The behaviour of the first two doublets is obvious enough: ordinary spin-orbit effects vanish for half closed shells such as $2 \mathrm{p}^{3}$, and if they seem to be splitting, so ${ }^{2} D^{o}$ by a slender wrongly ordered $0.04 \mathrm{~m} \mathrm{Ry}$, it is due to the spin-orbit term coupling among the three odd parity $J=3 / 2$ levels, while ${ }^{2} D_{5 / 2}^{0}$ and ${ }^{2} P^{o} / 2$ remain in place; two-body magnetic interaction inside ${ }^{2} \mathrm{D}^{\mathrm{o}}$ suffices to put things right in accord with observation as seen in the last two columns. The first even parity doublet ${ }^{2} \mathrm{D}$ reveals a more intricate interplay, not merely because finestructure splitting now involves ordinary spin-orbit coupling reduced by two-body effects (which would still yield the inverted doublet order): the term coupling with lower levels revert this trend in an expansion that is truncated at level 16, and it needs coupling with even parity $J=3 / 2$ and $5 / 2$ from higher $2 s^{2} 2 p^{2} 3 s$ terms for correct finestructure splitting: see bracketed entries.

Equally satisfactory for such a modest-and for the collisional application readily 
manageable - configuration expansion is the term separation. On including all terms up to configuration $2 s^{2} 2 p^{2} 3 d$, the first 35-computed levels appear in the observed order. While most of the target energies are in a reasonable agreement with observation, we employ experimental values in the diagonal elements of the $(N+1)$-electron Hamiltonian to ensure correct resonance positions relative to the target thresholds.

Wavefunctions $P_{n l}(r)$ are supplied to the collision code not up to RA, the $R$-matrix box radius, but truncated at $r_{\text {cut }}$, a radius beyond which BPRM can continue tabulating analytically as a QDT expansion along section 2.1-ss of Berrington et al (1995). If BPRM seems to reproduce fewer digits for the mean radius $\bar{r}$ of the $3 \mathrm{~d}$ orbital, look for the answer in the last column of table 2: an orbital merely used for correlation purposes need not extend to

Table 3. Term-coupling coefficients of the OII levels with $J=3 / 2$ in the ground configuration $2 s^{2} 2 p^{3}$ (the four odd parity correlation configurations show up in the last three digits, hence the off-diagonal differences-relative signs from superstructure); 1-magn and 2-magn as in table 1.

\begin{tabular}{|c|c|c|c|c|}
\hline \multicolumn{2}{|c|}{$J=3 / 2$} & \multirow[b]{2}{*}{$\mathrm{s}-\mathrm{O}$ angular factor } & \multicolumn{2}{|c|}{ term-coupling coefficient } \\
\hline level & component & & 1 -magn & 2-magn \\
\hline \multirow[t]{3}{*}{${ }^{4} S^{\circ}$} & ${ }^{4} S^{\circ}$ & 0.0 & 0.999990 & 0.999990 \\
\hline & ${ }^{2} \mathrm{D}^{\mathrm{o}}$ & 0.0 & +0.000032 & +0.000157 \\
\hline & ${ }^{2} \mathrm{P}^{\mathrm{o}}$ & 1.000000 & -0.004569 & -0.004365 \\
\hline \multirow[t]{3}{*}{${ }^{2} \mathrm{D}^{\circ}$} & ${ }^{4} S^{\circ}$ & 0.0 & -0.000122 & -0.000239 \\
\hline & ${ }^{2} \mathrm{D}^{\circ}$ & 0.0 & 0.999806 & 0.999823 \\
\hline & ${ }^{2} \mathrm{P}^{\circ}$ & 1.118034 & -0.019681 & -0.018829 \\
\hline \multirow[t]{3}{*}{${ }^{2} \mathrm{po}^{\mathrm{o}}$} & ${ }^{4} S^{\circ}$ & 1.000000 & +0.004568 & +0.004361 \\
\hline & ${ }^{2} \mathrm{D}^{\circ}$ & 1.118034 & +0.019681 & +0.018830 \\
\hline & ${ }^{2} \mathrm{P}^{\circ}$ & 0.0 & 0.999796 & 0.999813 \\
\hline
\end{tabular}

$\mathrm{RA}=21.52$, the value automatically set if all radial functions must decay below relative magnitude 0.002 , when differences reduce to 1 in the last digit for the valence and correlation orbitals. While all our orbitals are spectroscopic in character (also in mere correlation configurations), Bell and Hibbert (1990) chose contracted Slater-type orbitals $\overline{3} \mathrm{p}$ and $\overline{3} \mathrm{~d}$ whose mean radii $\mathrm{r}$ are thus markedly smaller: $2.0724 a 0$ and $1.2874 a 0$ respectively.

Unlike superstructure, the atomic structure code CIV3 (Hibbert 1975) allows for the selective term expansion according to coupling schemes. Perhaps the good ${ }^{2} D^{o}-{ }_{-}^{4} S^{o}$ separation of 0.2479 Ry by Bell et al owes something to their choice, looking less favourable for ${ }^{2} \mathrm{Do}$ with 0.3740 Ry and deteriorating to 0.38297 Ry when we follow their state descriptions. By and large our target energies look superior when their expansions are not truncated. Applied to BPRM the selection procedure requires subtle choices in equation (8). How McLaughlin and Bell made these selections is not clear from their outline.

The smallness of the term-coupling coefficients is significant, negating the claim that relativistic effects might play a crucial role in the line intensity ratios; see table 3 . It should be recalled that the second-order ordinary spin-orbit effects swamp those from two-body magnetic coupling in species of high ionization - as the basis of numerous Breit-Pauli calculations without two-body magnetic finestructure terms. In the current work it is their smallness, having made sure that level inversions have no adverse side effects when matching the target energies in STGH of BPRM to observation. Table 3 is also interesting in that the collision strengths to ${ }^{2} D^{o}$ and ${ }^{2} P^{o}$ are of 
the same order of magnitude so that the flux redistribution is well described by the TCCs alone.

In addition to the 16-target functions $\chi_{i}$, the CC expansion (8) of OII involves bound (e + OII) correlation functions $\Phi j$ comprising all possible combinations of OI configurations formed by one- and two-electron excitations from $2 \mathrm{~s}$ through $3 \mathrm{~d}$ orbitals. It is conveniently tested with bound-state boundary conditions: we obtain eigen energies for the three Or ground term levels $2 s^{2} 2 p^{4}\left({ }^{3} P_{2,1,0}\right)$ within $0.3 \%$ of the experimental values. We plan to further describe these calculations for the bound-bound and bound-free transitions of OI in a separate publication.

The BPRM intermediate coupling calculations for the 16 levels are carried out with partial waves $\ell \leq 15$ involving total $J \pi$ symmetries with $O \leq J \leq 12$, both even and odd parities. The partial wave summation ensures convergence for the forbidden transitions among all levels of the ground configuration. We had NRANG2 $=15 R$-matrix basis functions in the inner region, with a boundary $\mathrm{RA}$ at $17 \mathrm{Bohr}$ radii. The target orbital last point of inflection-see
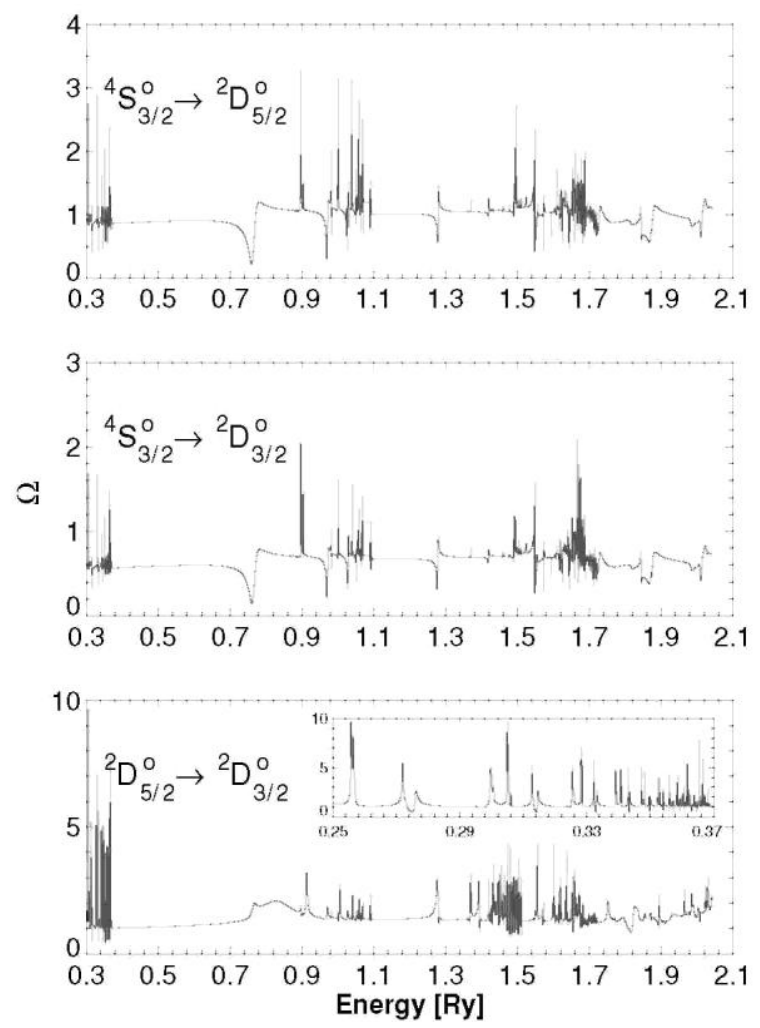

Figure 1. Collision strengths for the excitation of the ${ }^{2} D_{5 / 2,3 / 2}^{0}$ levels from the ground state ${ }^{4} \mathrm{~S}_{3 / 2}^{0}$ responsible for the formation of astrophysical important lines at $3729 \AA$ and $3726 \AA$ (top and middle panels, respectively). Note that the ratio of the collision strengths themselves at all energies is 1.5 throughout, showing absence of relativistic mixing and thereby determining the low-density limit to be the same. The collision strength ${ }^{2} D_{5 / 2}^{0}-{ }^{2} D_{3 / 2}^{0}$ for collisional redistribution due to electron impact between the two excited levels is shown in the bottom panel; the inset shows an expanded view of the near-threshold resonances. Whereas resonances do not play a major role in the excitation from the ground level, they are more significant for collisional mixing.

$r_{\text {infl }}$ in table 2-is a key guide to these choices, as the partial wave first point of inflection is analytically known as a function of $k$ and $\ell$. 


\section{Results and discussion}

We describe several sets of results that demonstrate the salient features of the collision strengths and parameters for applications. In addition we compare with the measured cross sections for the ${ }^{4} S^{0}-{ }^{2} D^{0}$ transitions.

\subsection{Collision strengths}

Figure 1 shows the collision strengths $\Omega\left({ }^{4} S_{32}^{0}-{ }^{2} D_{5 / 2,3 / 2}^{o}\right)$ from the ground level to the two finestructure ${ }^{2} D o$ levels, and the collision strength $\Omega\left({ }^{2} D_{5}^{0} \quad 12-{ }^{2} D_{3 / 2}^{0}\right)$ responsible for collisional mixing between them. The first thing to note is that the ratio of the former collision strengths is 6:4 throughout the energy range under consideration, including the resonant and the
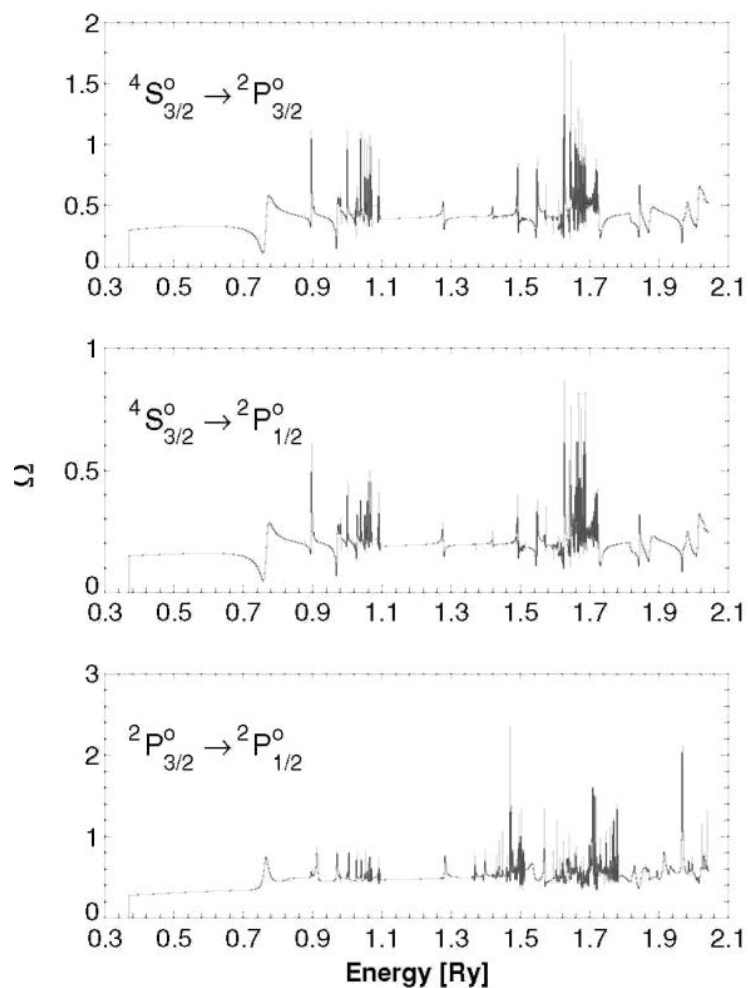

Figure 2. Collision strengths for the excitation of ${ }^{4} S_{3 / 2}^{0}-{ }^{2} P_{3 / 2,1 / 2}^{o}$ These also divide according to the ratio of statistical weights.

non-resonant regions. Therefore, no deviation from the $L S$ coupling values is seen. Secondly, resonances are not a major contributor to collisional excitation from the ground level. However, the collision strength $\Omega\left(D_{5 / 2}^{0}-{ }^{2} D_{3 / 2}^{0}\right)$ does have a significant contribution from resonances, particularly in the important near-threshold region. Interestingly, these figures are the first clear representation of these OII collision strengths. In an earlier work (P76a,b), the collision strengths were not plotted and were tabulated only at a few near-threshold values. In the more extensive MB98 paper, the collision strengths are plotted to $30 \mathrm{Ry}$; this results in the near-threshold region $E$ $\leq 1 \mathrm{Ry}$ as far too compressed to discern any resonance structures in detail. Therefore, it is not 
possible to directly compare the present collision strengths with MB98. The designation of the first set of Rydberg resonances in figure 1 is $\left({ }^{2} P^{o}\right) n \ell$. These are the most important resonances in the forbidden transitions at $T \approx 10^{4} \mathrm{~K}$. The higher ones begin with $\left({ }^{4} \mathrm{P}\right) n \ell$; exact identification depends on the closely spaced finestructure sub-levels. Similarly, one may obtain approximate designations relative to the target thresholds given in table 1 .

Figure 2 gives the collision strengths for excitation from the ground to the ${ }^{2} \mathrm{P}_{3 / 2,1 / 2}^{\mathrm{O}}$ levels. Again we find no evidence of redistribution of collision strength due to relativistic mixing, and the finestructure values divide according to statistical weights.

This is further borne by figure 3, which shows the four finestructure transitions within the ${ }^{2} D_{J}^{o}-{ }^{2} P_{J}^{o}$, multiplet. The algebraic finestructure to $L S$ collision strength ratios $\Omega(S L J-$ $\left.S^{\prime} L^{\prime} J^{\prime}\right) / \Omega\left(S L-S^{\prime} L^{\prime}\right)$ correspond to the non-relativistic ones given in table II of P76a (there is a minor deviation at some resonance energies owing to resolution).

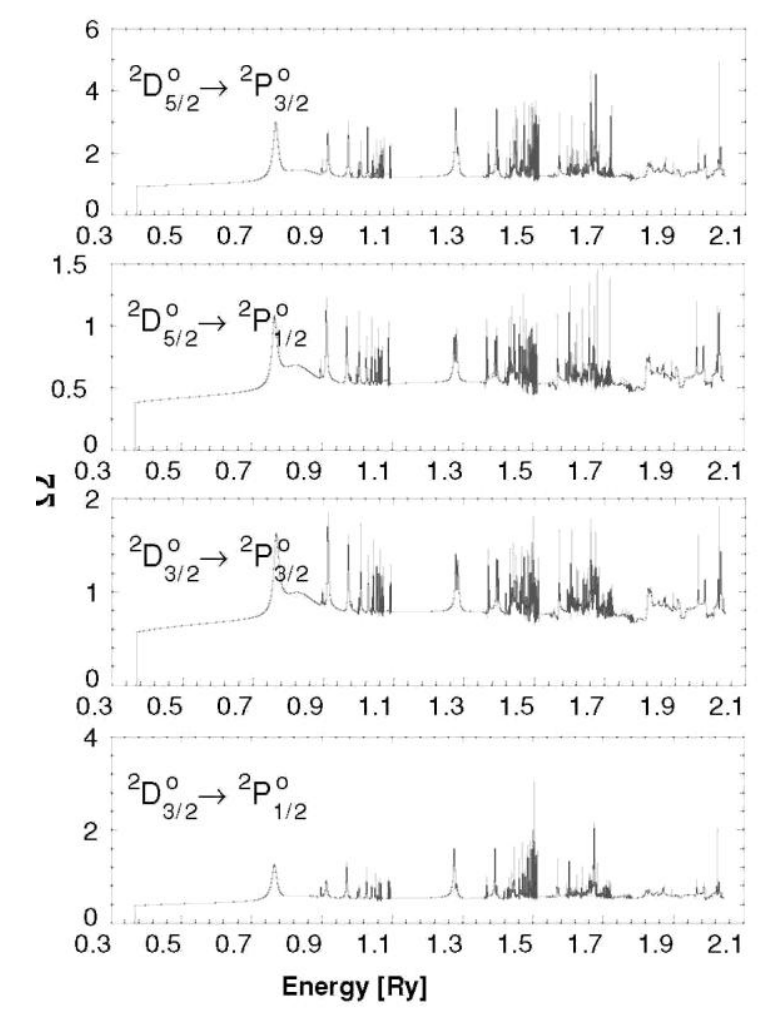

Figure 3. Collision strengths for the array of four transitions ${ }^{2} D_{5 / 23 / 2}^{0}-P_{z / 2,1 / 2}^{0}$. The ratios of the finestructure to $L S$-term collision strengths are approximately as given in the non-relativistic $L S$ coupling results of P76a,b.

Altogether these ten transitions are the ones responsible for the formation of the forbidden [OII] lines.

\subsection{Maxwellian-averaged effective collision strengths}

The Maxwellian-averaged or effective collision strengths $Y_{i j}(T)$ reveal the precise magnitude of differences with earlier works by P76a,b and MB98. Figure 4 shows $Y(T)$ for the ${ }^{4} S_{3 / 2}^{0}-{ }^{2} D_{5 / 2,3 / 2}^{0}$ transitions. The present results are slightly yet significantly higher than P76 for both transitions, 
though within $10 \%$. This is caused by including considerably more resonance structure. More importantly, the ratio of the two collision strengths is the same as in the earlier work.

However, the differences with MB98 are more pronounced, and especially for the ${ }^{4} \mathrm{So}-{ }^{2} \mathrm{D}{ }_{3 / 2}^{0}$ transition the MB98 values are nearly 30\% lower. It is this difference that largely accounts for the line intensity ratio $I$ (3729)/I (3726) in figure 4 Keenan et al (1999) to be higher by the same amount using the MB98 collision strengths. K99 quote $Y\left({ }^{4}\right.$ So $\left.-{ }^{2} \mathrm{D}^{0}{ }_{3 / 2}\right)=0.422$ from MB98, compared to 0.584 from P76a and 0.585 from Pradhan et al (2006) based on the present work. The two publications also differ significantly for other forbidden transitions from this investigation (figures 5 and 6). Whereas the differences with P76a,b are understandable in terms of the limited nature of the previous calculations, the differences with MB98 are inexplicable. Normally we should not deviate by more than about $10 \%$, since both are R-matrix calculations with similar eigenfunction expansions. Moreover, relativistic effects are included using the same set of BPRM codes.

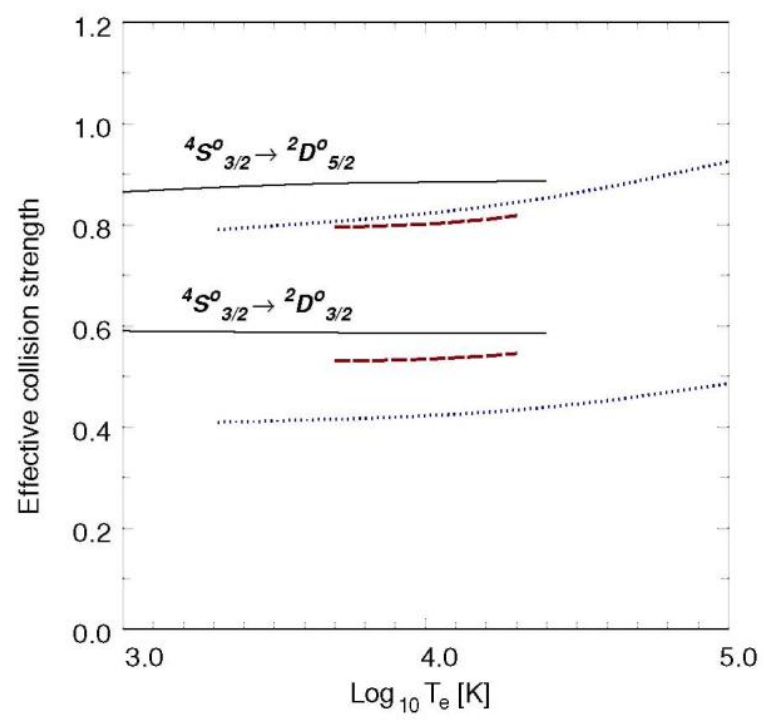

Figure 4. Maxwellian-averaged collision strengths $Y(T)$ corresponding to figure 1 (solid line), compared to the earlier calculations in P76a,b (dashed line) and MB98 (dotted line). The temperature dependence is much less pronounced than MB98, and the ratio is roughly constant at 1.5 


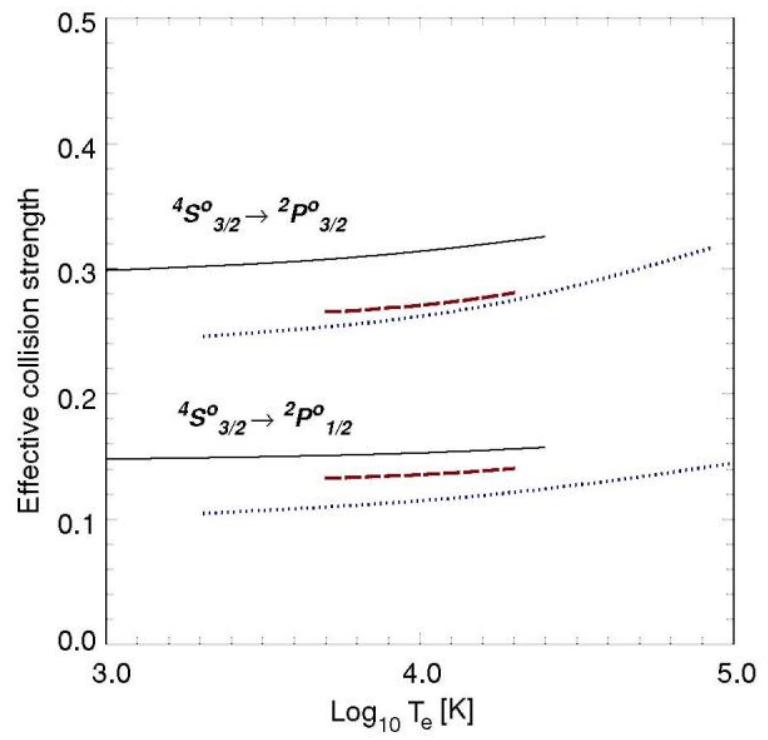

Figure 5. Maxwellian-averaged collision strengths corresponding to figure 2, with labels as in figure 4.

Because we delineate resonances better than P76a,b, which had limited resolution, the collisional mixing transitions ${ }^{2} \mathrm{D}_{5 / 2}^{\mathrm{o}}-{ }^{2} \mathrm{D}_{32}^{\mathrm{o}}$ and ${ }^{2} \mathrm{P}_{3 / 2}^{\mathrm{o}}{ }^{2} \mathrm{P}_{1 / 2}^{\mathrm{o}}$ shown in figure 7 differ significantly; so they do from MB98. 

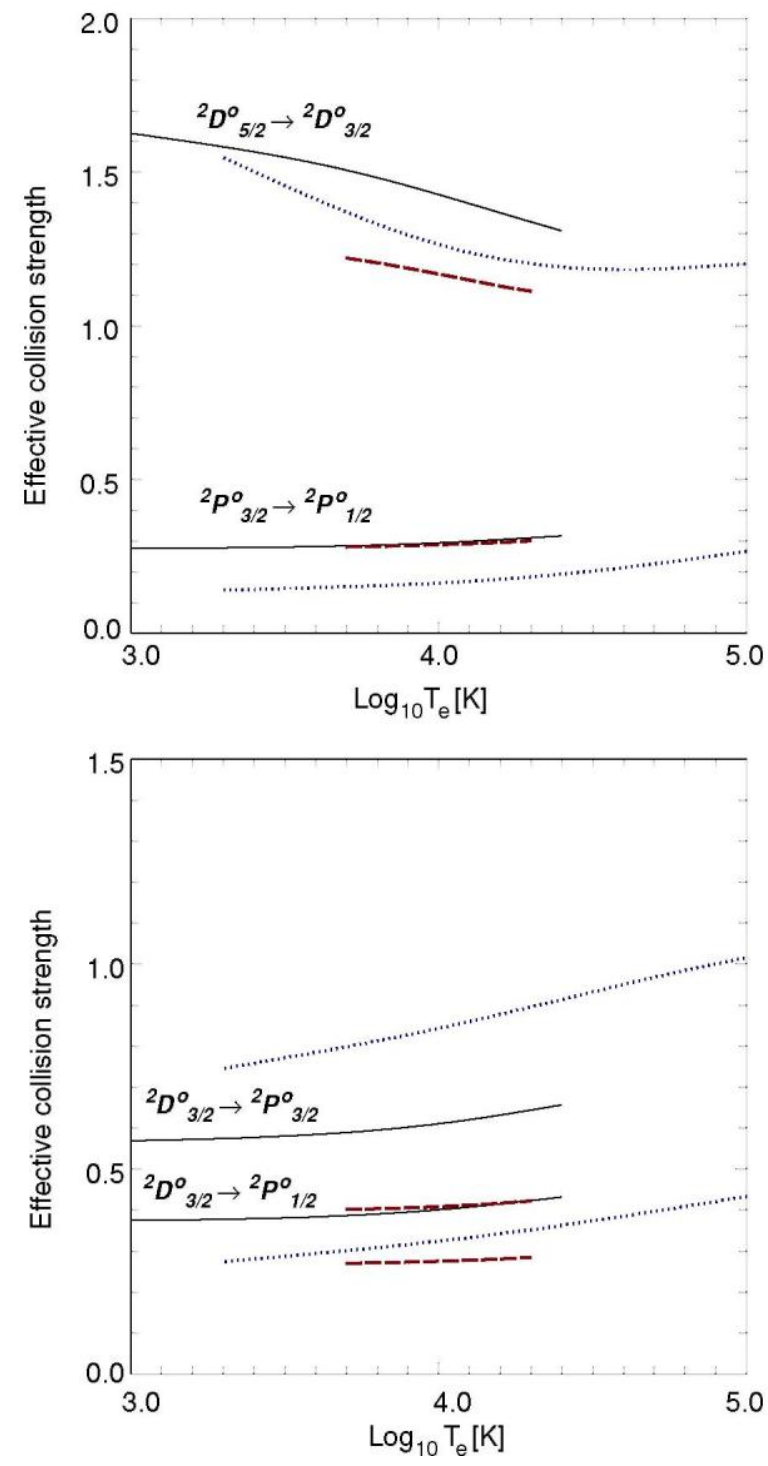

Figure 6. Maxwellian-averaged collision strengths for the four transitions in figure 3, with labels as in figure 4.

4.2.1. Comparison with experiment. The experimental group at the Jet Propulsion Laboratory has carried out absolute measurements of the cross sections for excitation of the ${ }^{4} \mathrm{~S}^{0}-{ }^{2} \mathrm{D}^{0}$ transition using the merged beam method (Zuo et al 1995). In figure 8 we compare the present-calculated cross sections with those in figure 8 of (Zuo et al 1995). Figure 8(a) shows the comparison with the detailed cross sections, and figure 8(b) shows the calculated cross sections convolved over the 250 $\mathrm{meV}$ experimental beam width. Though the experimental error bars for some points are as large as $50 \%$, it is noteworthy that the present theoretical results pass through nearly all measured values. In particular, the convolved cross section in figure 8 shows that the first point above threshold is within the error bars of the first-measured 


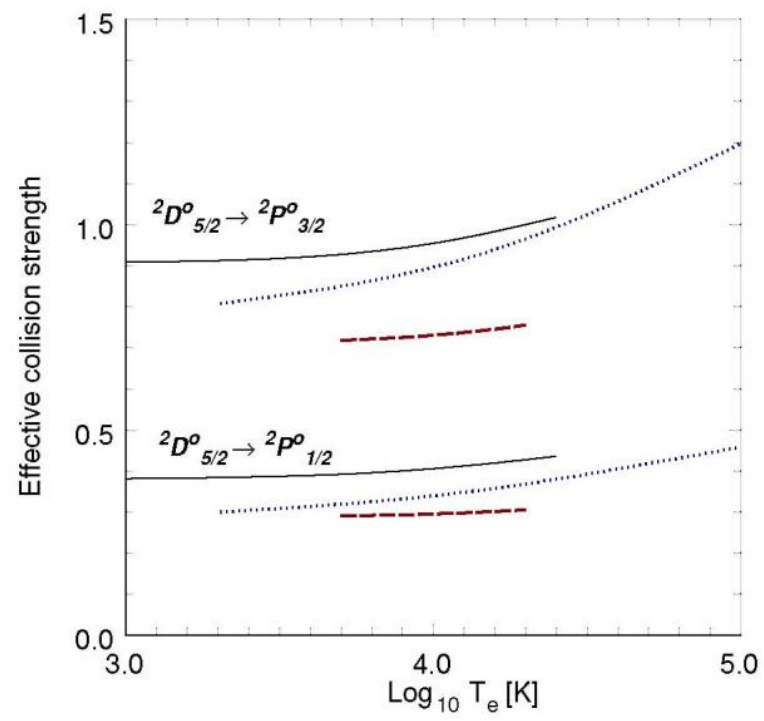

Figure 7. Maxwellian-averaged collision strengths for the collision mixing transitions between the finestructure levels ${ }^{2} \mathrm{D}_{52,32}^{0}$ and ${ }^{2} P_{32,1 / 2}^{o}$; labels as in figure 4 .

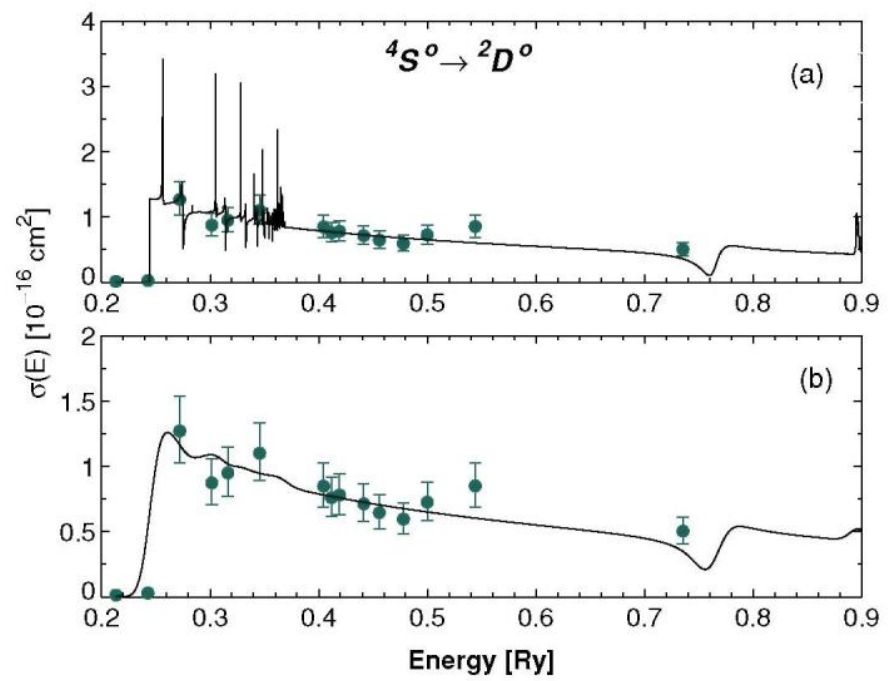

Figure 8. Comparison of the calculated electron impact excitation cross sections $0\left({ }^{4} \mathrm{So} \rightarrow{ }^{2} \mathrm{Do}\right)$ with the experimental measurements of Zuo et al (1995). Panel (a) shows the experimental values directly compared with the detailed cross sections, whereas panel (b) compares with the calculated cross sections convolved with the $250 \mathrm{meV}$ experimental beam distribution.

value. The important near-threshold cross sections as well as the subsequent fall-off with energy above $E\left({ }^{2} \mathrm{P}^{0}\right)=0.37 \mathrm{Ry}$ are in a very good agreement with the behaviour seen experimentally. However, differences in details remain for a few points; in particular at $0.55 \mathrm{Ry}$ there seems to be an unexplained rise in the experimental value: we expect the cross section to be monotonically decreasing up to the first resonance due to higher thresholds at $0.75 \mathrm{Ry}$.

\subsection{Line ratios}

In a recent paper (Pradhan et al 2006) we have discussed the astrophysical implications of the 
results presented in this detailed report. Although the precise source of the discrepancy with MB98 is not clear, the present effective collision strengths have largely reconfirmed the earlier results of P76a,b. The I (3729)/I (3726) line intensity ratio given by Pradhan et al (2006) satisfies the lowand high-density limits exactly: equations (5) and (6), respectively.

Pradhan et al (2006) also give the Maxwellian-averaged effective collision strengths $Y(T)$ for the ten transitions discussed herein, for the calculation of the forbidden [OII] line intensity ratios at a few temperatures, using the $A$-coefficients by Zeippen (1982).

\section{Conclusions}

The principal features of the present work are as follows.

- The collision strengths for the forbidden transitions in OII are not affected by relativistic effects. New BPRM calculations for electron impact excitation of the lowest ten forbidden transitions in OII have shown that the outstanding discrepancy between the observed line intensity ratios of [OII], and those calculated using the earlier results of McLaughlin and Bell (1998) are not due to relativistic effects, which are negligibly small.

- Further work is in progress on developing a more comprehensive target for OII to compute collision strengths for the allowed transitions.

\section{Acknowledgments}

This work was partially supported by the US National Science Foundation. Most of the computational work was carried out on the Cray X1 at the Ohio Supercomputer Center, Columbus /Ohio.

\section{References}

Bell K L, Burke P G, Hibbert A and Kingston A E 1989 J. Phys. B: At. Mol. Opt. Phys. 22 3197-204

Bell K L and Hibbert A 1990 J. Phys. B: At. Mol. Opt. Phys. 23 2673-85

Berrington K A, Eissner W B and Norrington P H 1995 Comput. Phys. Commun. 92 290-420

Burke P G, Hibbert A and Robb W D 1971 J. Phys. B: At. Mol. Phys. 4 153-61

Copetti M V F and Writzl B C 2002 Astron. Astrophys. 382 282-90

Crees M A, Seaton M J and Wilson P M H 1978 Comput. Phys. Commun. 15 23-83

Dopita M A and Sutherland R S 2003 Astrophysics of the Diffuse Universe (Berlin: Springer)

Drake G W 1971 Phys. Rev. A 3 908-915

Eissner W and Nussbaumer H 1969 J. Phys. B: At. Mol. Phys. 2 1028-43

Eissner W and Seaton M J 1972 J. Phys. B: At. Mol. Phys. 5 2187-98

Eissner W and Seaton M J 1974 J. Phys. B: At. Mol. Phys. 7 2533-48

Eissner W and Zeippen C J 1981 J. Phys. B: At. Mol. Phys. 14 2125-37

Hibbert A 1975 Comput. Phys. Commun. 9 141-72

Keenan F P, Aller L H, Bell K L, Crawford F L, Feibelman W A, Hyung S, McKenna F C and McLaughlin B M 1999 Mon. Not. R. Astron. Soc. 304 27-34

McLaughlin B M and Bell K L 1998 J. Phys. B: At. Mol. Opt. Phys. 31 4317-29

Osterbrock D E 1989 Astrophysics of Gaseous Nebulae and Active Galactic Nuclei (Mill Valley, CA: University Science Books)

Pradhan A K 1976a J. Phys. B: At. Mol. Phys. 9 433-43

Pradhan A K 1976b Mon. Not. R. Astron. Soc. 177 31-8

Pradhan A K, Montenegro M, Nahar S N and Eissner W 2006 Mon. Not. R. Astron. Soc. 366 L6

Scott N S and Taylor K T 1982 Comput. Phys. Commun. 25 347-87

Seaton M J 1987 J. Phys. B: At. Mol. Phys. 20 6363-78

Seaton M J and Osterbrock D E 1957 Astrophys. J. 12566 
Wang W, Liu X W, Zhang Y and Barlow M J 2004 Astron. Astrophys. 427 873-86

Wenåker I 1990 Phys. Scr. 42 667-84

Zeippen C J 1982 Mon. Not. R. Astron. Soc. 198 111-25

Zuo M, Smith S J, Chutjian A, Williams I D, Tayal S S and McLaughlin B M 1995 Astrophys. J. 440 421-9 